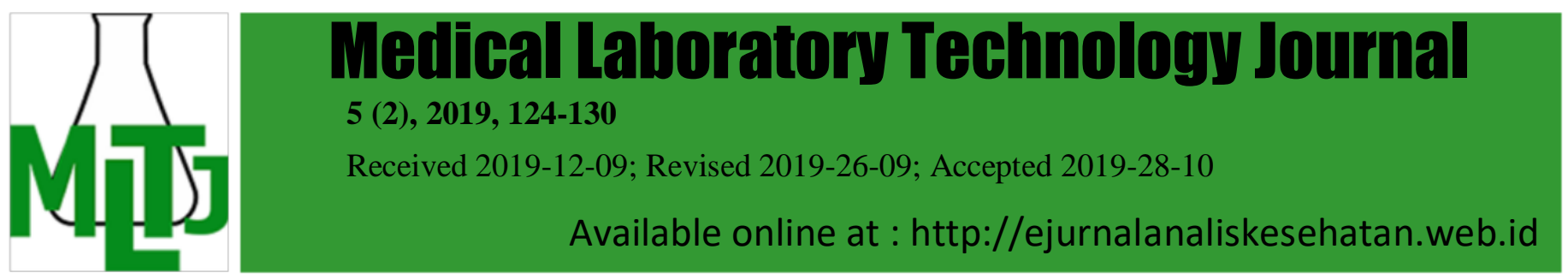

\title{
Detection of Mycobacterium tuberculosis by Microscopic Technique of Sputum AFB and ICT (Cocktail Antigen) TB
}

\section{${ }^{*}$ Retno Martini Widhyasih, Annisa Husnun Hanifah, Chairlan, Dewi Inderiati}

Medical Laboratory Technology, Health Polytechnic of Jakarta III Ministry of Health Jalan Arteri JORR Jatiwarna, Kecamatan Pondok Melati, Bekasi, 17415, Indonesia.

E-mail : retnomartiniw@gmail.com

DOI: $10.31964 / \mathrm{mltj} . v \%$ vi\%i.246

\begin{abstract}
Tuberculosis is an infectious disease caused by Mycobacterium tuberculosis. The diagnosis of pulmonary TB in general still relies on microscopic examination with a sputum smear for Acid Fast Basil (AFB). Microscopic examination to detect $M$. tuberculosis has high specificity, but the sensitivity is $35-70 \%$. The sputum culture method is the gold standard for the diagnosis of pulmonary TB but requires a long time, which is 6-8 weeks. In recent years ICT (Immuno Chromatography Test) has been introduced to identify potential and useful TB antigens to help diagnose pulmonary TB. Antigens detected were ESAT-6, CFP-10, CFP-21, and MPT-64. This study aims to compare the results of the examination of specimens of suspect TB sputum with the ICT method and AFB. This study based on observational with analytic design cross-sectional on 56 samples of Stored Biological Material suspects TB that were examined by the AFB method and the ICT method (Cocktail Antigen). Statistical test results Fisher Exact $(\alpha=0.05)$ showed no difference between microscopic examination of smear sputum and ICT (Cocktail Antigen) TB $(p=1,000)$. Nevertheless, the use of ICT reagents for the diagnosis of pulmonary TB still needs to be further investigated, especially to find out the exact causes of false positive and false negative reactions.
\end{abstract}

Keywords: ICT (Cocktail Antigen); smear microscopic sputum; Mycobacterium tuberculosis

\section{INTRODUCTION}

Tuberculosis (TB) is an infectious disease caused by the bacterium Mycobacterium tuberculosis (M. tuberculosis) (WHO, 2018). This bacterium usually attacks the lung organs (pulmonary TB) but can also attack other organs such as the kidneys, spine, and brain (extra-pulmonary TB) (CDC, 2016). In 2017, WHO estimated the number of TBs to infect around 9-11.1 million people in the world (around 5.8 million men, 3.2 million women, and 1 million children) and caused 1.3 million cases of death (WHO, 2018). Based on the indicator Case Notification Rate (CNR), in 2015, TB cases in Indonesia recorded 130 cases per 100,000 population. In 2016, TB cases increased to 139 cases per 100,000 population. This number of cases increase lasts until 2017, reaching 161 cases per 100,000 population (Ministry of Health: InfoDatin, 2018).

TB cases in Indonesia are the third rank after India and China in 2017 (WHO, 2018). Data TB of DKI Jakarta Province in 2017 obtained 35,733 (Ministry of Health, 2017). The number of suspected pulmonary TB in DKI Jakarta in 2017 was 36,998 patients, compared to 2016 as many as 55,503 patients. The number, 12,880 of them are new TB positive patients; there is an increase in TB cases positive new cases compared to 2016 by 7,302 patients. East Jakarta is one of the regions with

Corresponding Author: Retno Martini Widhyasih

Medical Laboratory Technology, Health Polytechnic of Jakarta III Ministry of Health

Jalan Arteri JORR Jatiwarna, Kecamatan Pondok Melati, Bekasi, 17415, Indonesia.

Email: retnomartiniw@gmail.com 
the highest number of positive pulmonary TB cases in DKI Jakarta of 4,176 cases (DKI Jakarta Health Office, 2017). These cases strike a lot in the productive adult age group, and TB can also strike in any age group (WHO, 2018). The results of (Basic Health Research,2013) concluded that as people age, the prevalence of TB is higher.

This is because the possibility of TB reactivation and duration of TB exposure is longer than the age group below. Characteristics of the population in low education and not working will increase the prevalence of TB. The mortality rate for TB sufferers in DKI Jakarta is 4.48 per 100,000 population (DKI Jakarta Provincial Health Office, 2017).

A TB diagnosis can be established, including clinical examination, laboratory examinations, and investigations. In laboratory tests, there are AFB, TB Molecular Rapid Tests (TCM), and culture, while the radiological examination is supporting (Permenkes No. 67, 2016). In developing countries still relying on microscopic examination with sputum smear for Acid Fast Basil (AFB) (Fennelly et al., 2012). AFB works for diagnosis are carried out because they are the simplest, fastest, and cheapest (Ministry of Health, 2012). The proportion of microscopic examination to detect $M$. tuberculosis is often low as $20-30 \%$ of all cases (WHO, 2009) Microscopic examination requires around 5,000-10,000 bacilli per milliliter of sputum to be found to be positive smear, so culture checks are needed to distinguish TB patients from Non Tuberculosis Mycobacterial (NTM) patients (WHO, 2009; Ministry of Health RI, 2012).

Until now, the method used for TB diagnosis still has many obstacles, especially in developing countries, to make TB diagnoses fast and accurate. Therefore a TB diagnosis method that is fast, accurate, and affordable is needed (Liu, Z. et al., 2016). In recent years, there has progressed in identifying potential and useful antigens to help diagnose pulmonary TB. Gen Region of Different (RD)13 acts as a virulence gene possessed by $M$. tuberculosis because it not found in $M$. bovis Bacillus Calmette-Guerin (BCG) (Wang et al., 2013, Subroto, H. et al., 2017). Serodiagnostic test research by Goyal, B. et al. (2014) combines specific antigens present in TB germs. Specific antigens (Antigen Cocktail) include ESAT-6, CFP-10, CFP-21, and MPT-64. It aims to study the usefulness of B cell epitope-based peptides from RD1 (ESAT-6, CFP-10) and RD2 (CFP-21, MPT-64, with indirect ELISA technique). In this study, we used a simpler and faster technique called ICT technique. This study was to determine the differences in the results of examinations between ICT (Cocktail Antigen) and smear in sputum specimens of patients who suspect TB.

\section{MATERIAL AND METHOD}

This study used analytic observational techniques using a design crosssectional conducted on 56 samples from February to March 2019 obtained from the Jatinegara District Health Center located in Jatinegara, East Jakarta, DKI Jakarta. Microscopic examination of AFB sputum and ICT (Cocktail Antigen) held at the Bacteriology Laboratory of the Medical Technology Building Laboratory of the Health Ministry of Health, Jakarta III. This study was approved by the Health Research Ethics Commission of the Health Ministry of Health III Jakarta with the letter-number KEPK-PKKJ3 / 27 / II / 2019 before data processing was carried out. The data obtained were analyzed by Fisher Exact test $(\alpha=0.05)$. 


\section{Collection of Specimens}

The inclusion criteria in this study include; good sputum specimens, namely: sputum volume 3-5 mL, sputum conditions contain several particles or slightly thick and slimy, and sometimes purulent. Sputum specimens that contain much saliva (saliva), bloody sputum, and patients with TB who have or are undergoing treatment are not part of the inclusion criteria in this study.

In this study carried out on 56 sputum specimens from patients suspect TB examined in the Jatinegara District Health Center. It said that suspect TB is the presence of clinical symptoms felt by the patient, such as a history of tuberculosis, having been exposed or not to TB patients. Other symptoms such as prolonged cough for $>2-3$ weeks, bloody cough, chest pain, reduced appetite, sudden weight loss, sudden cold sweats at night, fever, and fatigue.

\section{Microscopic Examination of AFB sputum}

Sputum Purulent from patients suspects TB collects two types of sputum, namely morning sputum (A) and sputum when / after a morning bath / after breakfast (B). An outward appearance of AFB made into two slides (one slide A and one slide B). Outward appearance made with a technique coiling with a size of $2 \times 3 \mathrm{~cm}$, then the Outward appearance staining with the Ziehl-Neelsen coloring technique. After dry and colored by Ziehl-Neelsen color, M. tuberculosis (red bacilli bacteria) is observed with a microscope Olympus CX-21 type with 1000x magnification.

\section{Examination of ICT (Cocktail Antigen) TB}

Examination antigens on these reagents were using methods immunochromatography (ICT). Reagent test JD Biotech Cat No: JTBBAG-02 (Card format) contains monoclonal antibody mouse anti-TB labeled with gold colloid is used to detect antigens ESAT-6, CFP-10, MPT-64, and phiRv1 in sputum. Nitrocellulose membranes consist of "Test band / (T)" and "Control band / (C)." In the test, band coated rabbit-anti-TB antibodies secreted antigen protein, and the $\mathrm{C}$ band coated with goat- anti-mouse IgG antibody. If there is an antigen, the conjugate antibody-dye will bind TB antigen so that it will form an antigen-antibody complex.

\section{Pre-treatment the samples}

A sample buffer is used to help dilute the purulent sputum. It also helps maintain the quality of the protein in the sample, helps release epithelial cells, and helps to eliminate bacteria that are not the type of Mycobacterium. The sample buffer mixed with sputum with a ratio of $1: 3$, one part sputum, and three parts sample buffer. Then the mixture is vortexed for 30 seconds. The purpose of the vortex is to make the mixture is homogenized entirely and facilitates the process of separation between proteins from bacteria $M$. tuberculosis by cell debris. After being vortexed, the mixture incubated at room temperature for 60 minutes. The goal is so that disrupting cell debris can be deposited entirely and not splattered from mixed droplets.

\section{Identify samples}

The pipette of 3-4 drops (60-80 $\mu \mathrm{L})$ from the resulting supernatant, then drops them in the area test band (T). Positive results indicate a red line formed in the area "C" and "T." Negative results indicate a red line in the " $\mathrm{C}$ " area. The invalid results only form on the "T" red line, or no red lines formed in either area. 


\section{RESULTS AND DISCUSSION}

Research has been carried out Differences in the Results of Examination Mycobacterium tuberculosis Between the ICT Method (Cocktail antigen) and the Microscopic Sputum Examination Method of AFB. The results are as follows:

Table 1. Cross Tabulation Results of Microscopic Examination of AFB Sputum and ICT (Cocktail Antigen) TB

\begin{tabular}{|c|c|c|c|c|c|}
\hline \multirow[t]{2}{*}{ Inspection Variable } & & \multicolumn{2}{|c|}{$\begin{array}{l}\text { Microscopic } \\
\text { AFB Sputum }\end{array}$} & \multirow{3}{*}{$\begin{array}{c}\text { Total } \\
8 \\
(14,3 \%)\end{array}$} & \multirow{2}{*}{$\begin{array}{c}\mathrm{p} \\
\text { Value* }\end{array}$} \\
\hline & & Positive & Negative & & \\
\hline $\begin{array}{l}\text { ICT Examination } \\
\text { (Cocktail Antigen) }\end{array}$ & Positive & $\begin{array}{c}1 \\
(1,8 \%)\end{array}$ & $\begin{array}{c}7 \\
(12,5 \%)\end{array}$ & & 1,000 \\
\hline TB & Negative & $\begin{array}{c}9 \\
(16,1 \%)\end{array}$ & $\begin{array}{c}39 \\
(69,6 \%)\end{array}$ & $\begin{array}{c}48 \\
(85,7 \%)\end{array}$ & \\
\hline Total & & $\begin{array}{c}10 \\
(17,9 \%)\end{array}$ & $\begin{array}{c}46 \\
(82,1 \%)\end{array}$ & $\begin{array}{c}56 \\
(100 \%) \\
\end{array}$ & \\
\hline
\end{tabular}

*statistical test Fisher Exact obtained $p$-value $(1,000)$ value is greater than alpha value $(\alpha)=(p>0.05)$. That is, there is no difference in results between the microscopic examination of smear sputum and ICT (Cocktail Antigen) TB.

\section{Microscopic examination of smear sputum}

In table 2., the results of the microscopic examination of AFB sputum and ICT (Cocktail Antigen) TB were $1(1.8 \%)$ of 8 positive samples on microscopic examination of AFB sputum and ICT (Cocktail Antigen) TB. Whereas 7 (12.5\%) of the eight samples were positive for a microscopic smear but negative for examination (Cocktail Antigen) TB. Results $39(69.6 \%)$ of 48 negative samples on microscopic examination of AFB sputum and ICT (Cocktail Antigen) TB. Whereas 9 $(16.1 \%)$ of 48 samples were negative for microscopic examination of AFB sputum but positive for examination (Cocktail Antigen) TB.

Sputum examination is important because, with the discovery of AFB germs, the diagnosis of TB can be ascertained. Besides, sputum examination can also provide an evaluation of the treatment that has given.

Detection with AFB staining chosen because of economic reasons, but the limitations of this method greatly influenced by several external factors such as the quality of the staining and examiner's expertise, resulting in inaccurate examinations. The best standard in examining TB is a bacterial culture, but this stage has a weakness because it is considered too long in giving results (Nazarudin, M. et al., 2012). 


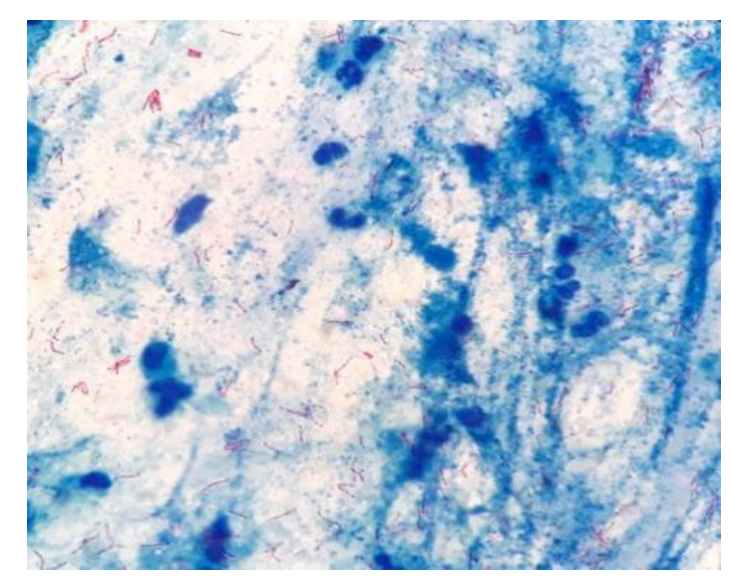

Figure 1. Visualization of bacteria M. tuberculosis with Ziehl-Neelsen staining at 1000x (Private Collection)

\section{ICT Inspection (Cocktail Antigen) TB}

At this time, there is a reagent that can detect $M$. tuberculosis in a fast way, namely examination of $M$. tuberculosis Antigen (TB Ag) Rapid Test, JD Biotech. This examination is a serological test of the ICT method. Reagent test contains monoclonal antibody mouse anti-TB labeled with gold colloid is used to detect antigens ESAT-6, CFP-10, MPT-64, phiRv1 in sputum. Nitrocellulose membranes consist of "Test band" and "Control band." In-band tests coated with rabbit-anti-TB secreted antibody-antigen protein and Control band coated with goat anti-mouse IgG antibody (JD Biotech Manual Kit Instruction, 2019).

From the results of the study, as many as 9 (16.1\%) samples gave negative ICT (Cocktail Antigen) TB results but a positive result in AFB sputum examination. This result is a false negative ICT examination of (Cocktail Antigen) TB. The possibility of a false negative cause in this study is that ICT reagents cannot detect a cocktail of antigens TB in sputum. Another possibility is that the samples in these two tests contain the cocktail antigen TB but are not detected because the levels are below the minimum detection value of ICT. ICT checks have a minimum level of sensitivity (sensitivity) that will give positive results on ICT. However, the lack of this kit instruction manual does not explain the sensitivity of the ICT reagent.

The study conducted by Toihir (2011), false negatives can be caused by strains that have gene mutations in antigens $M$. tuberculosis that might cause incomplete protein production. Also, other possibilities can be caused by the influence of the ICT strip storage temperature.

The difference in recombinant antibody antigen-capture will also affect the results. This study uses the ICT reagents of the JD Biotech (China) brand, thus allowing for differences in M. tuberculosis strains between China and Indonesia. The study of Haryanto (2015) explains that there are differences in the strains of Mycobacterium between West Java and East Nusa Tenggara (NTT). The Beijing genotype in West Java is $33 \%$, while NTT is only $14 \%$ and is more genotypically dominant than East Africa-India (EAI) and Latin America and Mediterranean (LAM) as much as $33.3 \%$ and $20 \%$. However, the two genotypes in West Java were $6.2 \%$ and $8.7 \%$. Sasmono et al. (2012) reported in Makassar, South Sulawesi, the dominant genotype of EAl was $30 \%$.

Based on table 1. there were 7 (12.5\%) samples (Cocktail Antigen) positive TB but on microscopic examination of AFB sputum. This is a possibility of false positives from examination (Cocktail Antigen) TB. Research by Subroto et al. (2017) false-positive results from ICT (Cocktail antigen) TB can occur if cross-reaction with 
MOTT (Mycobacterium Other Than Tuberculosis) which has an encoding gene ESAT-6 like protein and CFP-10 like protein. This means that in these organisms there are similarities between antigens and $M$. tuberculosis. These organisms include $M$. kasasii, $M$, szulgai, $M$. gastri, $M$. flavescens, and $M$. smegmatis (Turbawaty et al. 2017).

However, in a study conducted by Gustiani et al. (2014), ICT TB antigen examination with culture examination as a gold standard has a high validity to sensitivity (95.7\%) and specificity (87.2\%).

A limitation of this study is the difficulty in collecting homogeneous samples (samples that are purulent). Besides, further research needs to done by using samples of TB suspects patients not only for AFB examination but also using culture/culture samples so that the bias results are more accurate.

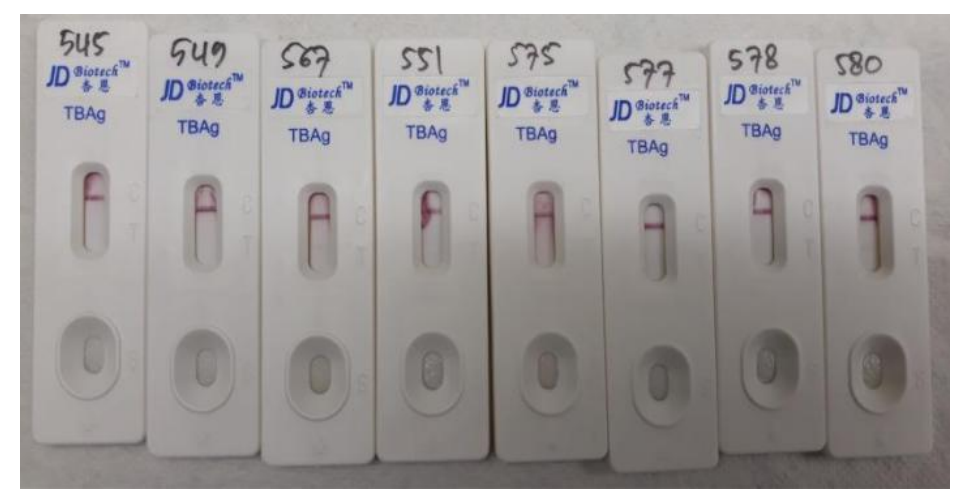

Figure 2. Results of antigen examination with ICT (Cocktail Antigen)

TB (Private Collection)

\section{CONCLUSION}

Based on the results of this study, there is no difference between the microscopic examination of AFB sputum and ICT (Cocktail Antigen) TB. However, the use of ICT reagents for the diagnosis of pulmonary TB must still be investigated further, especially to determine the exact cause of false positive and false negative reactions.

\section{REFERENCES}

Basic Health Research. (2013). Health Research and Development Agency. Republic of Indonesia Ministry of Health.

Bella, Goyal., Kumar, K., Gupta, D., Agarwal, R., Latawa, R., Sheikh, J.H., \& Verma, I. (2014). The utility of B-Cell Epitopes Based on Peptides of RD1 and RD2 Antigens for Immunodiagnosis of Pulmonary Tuberculosis. Diagnostic

Microbiology and Infectious Disease, 4, 391-397. http://dx.doi.org/10.1016/j.diagmicrobio.2013.12.018.

CDC (2016) Basic TB Fact - Understanding MTB.

DKI Jakarta Provincial Health Office. (2017). Health Profile of the DKI Jakarta Province in 2017.

Fennelly, K. P., Morais, C. G., Hadad, D. J., Vinhas, S., Dietze, R., \& Palaci, M. (2012). The Small Membrane Filter Method of Microscopy to Diagnose Pulmonary Tuberculosis. Journal of Clinical Microbiology, 50(6), 2096-2099. DOI:10.1128/JCM.00572-12

Gustiani, N., Parwati, I., Tjandrawati, A., \& Lismayanti, L. (2014). Validitas

Pemeriksaan Complex Specific Antigen Mycobacterium tuberculosis Region of 
Difference 1-3 Metode Rapid Immunochromatography Pada Sputum Penderita Tuberkulosis Paru. MKB, 6(4), 241-246.

Haryanto, Budi. (2015). Benefits of MPT64 TB Ag Immunocromatography Test for Differentiation of Mycobacterium tuberculosis Complex with Complex NonTuberculosis Mycobacterium. Thesis. Faculty of Medicine, University of Indonesia. Jakarta.

JD Biotech Instruction Kit Manual. (2019). M. Tuberculosis Antigen (TB Ag) Rapid Test.

Liu, Zhiqiang., Qie, Shuang., Li, Lili. Et al. (2016). Identification of Novel RD1 Antigens and Their Combinations for Diagnosis of Sputum Smear- / Culture + TB Patients. BioMed Research International, 2016, 1-10. http://dx.doi.org/10.1155/2016/7486425

Ministry of Health of the Republic of Indonesia. (2012). Technical Guidelines for Mycobacterium tuberculosis Culture, Identification, and Sensitivity Test in Solid Media.

Ministry of Health of the Republic of Indonesia. (2017). Health Profile Data and Information for 2017. Ministry of Health of the Republic of Indonesia.

Ministry of Health of the Republic of Indonesia. (2018). Tuberculosis: InfoDATIN 2018, InfoDATIN 2018 Ministry of Health of the Republic of Indonesia.

Nazarudin, M., Nugraha, J., \& Aryati. (2015). Diagnostic Value for TbAg and MPT64 Rapid Tests with Culture as the Gold Standard. Biosains Postgraduate Journal, 17(3), 1-16. http://dx.doi.org/10.20473/bsn.v17i3.Y2015.10790

Regulation of the Minister of Health (Permenkes) of the Republic of Indonesia No. 67. (2016). Ministry of Health of the Republic of Indonesia. 60-62.

Sasmono, R.T, Massi, M.N, Setianingsih, T.Y, Wahyuni, S., Anita., Malik, H., Yusuf, I,. . . Phyu, S. (2012). Heterogeneity of Mycobacterium tuberculosis Strains in Makassar, Indonesia. International Journal of Tuberculosis Lung Disease, 11(16), 1441-1448. http://dx.doi.org/10.5588/ijtld.12.0055

Subroto, H., Parwati, I., Turbawaty, D.K., \& Alisjahbana, B. (2017). Gambaran Validitas Pemeriksaan Complex Specific Cocktail Antigen Mycobacterium tuberculosis (ESAT-6, CFP-10, MPT-64) Metode Rapid Immunochromatography pada Bahan Pemeriksaan Sputum dan Serum

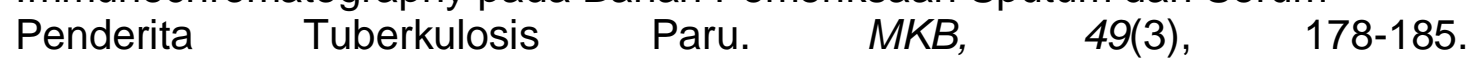
http://dx.doi.org/10.15395/mkb.v49n3.1120.

Toihir, Al-Habib Omar. Et al. (2011). Validation of the Immunochromatographic Assay Kit for the Identification of the Mycobacterium tuberculosis complex. Mem Inst Oswaldo Cruz, 106(6), 777-780. http://dx.doi.org/10.1590/S007402762011000600022

Turbawaty, D.K., Sugianli, A.K., Soeroto, A.Y., et al. (2017). Comparison of the Performance of Urinary Mycobacterium tuberculosis Antigens Cocktail ( ESAT 6, CFP10, and MPT 64) with culture and Microscopy in Pulmonary Tuberculosis Patients. International Journal of Microbiology, 2, 2-7 https://doi.org/10.1155/2017/3259329

Wang, S., et al. (2013). Mycobacterium tuberculosis Region of Difference (RD) 2 Antigen Rv1985c and RD11 Antigen Rv3425 Have the Promising Potential to Distinguish Patients with Active tuberculosis from M.bovis BCG-vaccinated Individuals. Clinical and Vaccine Immunology. 20(1), 69-76 DOI: 10.1128/CVI.00481-12

WHO. (2009). TB Diagnostics and Laboratory Services Information Note. pp: 1-7.

WHO. (2018). Global Tuberculosis Report. pp: 1-4, 188-189. 